\title{
Stability of the Forest Evolution System with Growth Functions
}

\author{
Bai Huihui \\ Department of Applied Mathematics, College of Science \\ Zhejiang University of Technology \\ Hangzhou, China
}

\begin{abstract}
This paper discusses the dynamical model of the Forest Evolution System, and the Existence and Uniqueness of the equation are have been proved by applying the theory of integral equation. Furthermore, we discussed the stability of the solution by applying the stability theory of Lyapunov.
\end{abstract}

Keywords-- forest development system; Integral equation; Operator Semi-groups; stability.

\section{INTRODUCTION}

Using mathematical models to study the Forest Evolution System has become a worldwide research hotspot in recent years, especially on considering the forest age factor and studying the forest evolution model described by partial differential equations. Reference [1] has discussed stationary forest development system and its properties, as well as the existence and uniqueness of the solution has been proved by using semigroup theory, reference [2] has given a kind of nonlinear forest development equations and discussed the properties of its solution, reference [3] has discussed the existence and uniqueness of solutions of a class of nonstationary forest development equations by using functional analysis and the fixed point principle of integral equation theory, reference [4-6] have discussed the existence and uniqueness of solutions of a class of stationary nonlinear development system with environmental impact factors. This article discusses the nonstationary forest evolution equations with growth functions, proves the existence and uniqueness of solutions of the nonstationary forest development system above by using the operator theory and integral equation theory, and discusses the stability of the equation in the sense of Lyapunov to get the stability and asymptotic stability sufficient conditions of the system.

This article mainly studies the nonstationary forest development system model as follows:

$$
\left\{\begin{array}{lc}
\frac{\partial p(r, t)}{\partial t}+\frac{\partial}{\partial r}(\varphi(r) p(r, t))=-\mu(r, t) p(r, t), & t, r>0 \\
p(r, 0)=p_{0}(r), & r \geq 0 \\
p(0, t)=v(t) \beta(t) \int_{0}^{Q_{m}} \mu(r, t) p(r, t) d r, & t \geq 0
\end{array}\right.
$$

Where, $p(r, t)$, which is called age-dependent forest area distribution, denotes the area of forest age $r$ at time $t, \varphi(r)$ denotes growth function, $p_{0}(r)$ is the initial condition. $\beta(t)$ denotes the ratio of the area of afforestation updating and mining (natural death elimination and artificial cut) at

\author{
Wang Dingjiang ${ }^{\mathrm{T}}$ \\ Department of Applied Mathematics, College of Science \\ Zhejiang University of Technology, Hangzhou, China \\ TEmail: wangdingj@126.com
}

time $t, v(t)$ denotes survival rate, $\mu(r, t)$ denotes dissipation rate, $Q_{m}$ is the max age.

\section{THE EXISTENCE AND UNIQUENESS OF SOLUTIONS}

In order to prove the existence and uniqueness of the solutions of system (1), we have to make the assumptions for the parameters of the system as follows $(\mathrm{H})$ :

(1). $v(t) \beta(t) \in C[0, T]$, where $T$ is an arbitrary fixed positive integer;

(2). $\varphi(r) \in C\left[0, Q_{m}\right]$ is a Monotone continuous function;

(3). $p_{0}(r) \in C\left[0, Q_{m}\right]$ and $p_{0}(0)=v(0) \beta(0) \int_{0}^{Q_{m}} \mu(r, 0) p(r, 0) d r$

(4). $\mu(r, t) \in C\left([0, T] \times\left[0, Q_{m}\right]\right), \mu(r, t)>0$.

Here we used the operator theory and integral equation to prove the existence and uniqueness of the solutions of system (1).

First of all, we used the characteristic line method in reference [4] to get the following solution of equation (1) :

$$
p(r, t)= \begin{cases}\varphi^{-1}(r) \varphi(r-\varphi(r) t) p_{0}(r-\varphi(r) t) & \\ \cdot \exp \left[-\int_{0}^{t} \mu(\varphi(r) \tau+r-\varphi(r) t, \tau) d \tau\right], & \frac{r}{\varphi(r)}>t \\ \varphi^{-1}(r) \varphi(0) p\left(0, t-\frac{r}{\varphi(r)}\right) & \\ \cdot \exp \left[-\int_{t-\frac{r}{\varphi(r)}}^{t} \mu(\varphi(r) \tau+r-\varphi(r) t, \tau) d \tau\right], & \frac{r}{\varphi(r)} \leq t\end{cases}
$$

We assume that the value of the functions $p(0, t), p(r, 0), \mu(t, r)$ is zero outside the domain, then we can get the general form of solution as follows:

$$
\begin{aligned}
p(r, t)= & \varphi^{-1}(r) \varphi(r-\varphi(r) t) p_{0}(r-\varphi(r) t) \\
& \cdot \exp \left[-\int_{0}^{t} \mu(\varphi(r) \tau+r-\varphi(r) t, \tau) d \tau\right] \\
+ & \varphi^{-1}(r) \varphi(0) p\left(0, t-\frac{r}{\varphi(r)}\right) \\
& \cdot \exp \left[-\int_{t-\frac{r}{\varphi(r)}}^{t} \mu(\varphi(r) \tau+r-\varphi(r) t, \tau) d \tau\right]
\end{aligned}
$$

Second, we convert system (1) to integral equation and discuss the existence and uniqueness of solutions.

From the boundary conditions of the system(1),

we can get 


$$
\begin{aligned}
p\left(0, t-\frac{r}{\varphi(r)}\right)= & v\left(t-\frac{r}{\varphi(r)}\right) \beta\left(t-\frac{r}{\varphi(r)}\right) \\
& \cdot \int_{0}^{Q_{m}} \mu\left(s, t-\frac{r}{\varphi(r)}\right) p\left(s, t-\frac{r}{\varphi(r)}\right) d s
\end{aligned}
$$

Putting the (4) into (3) we can get the following integral equation:

$$
p(r, t)=\int_{0}^{\varphi(r) t} K(r, t, s) p\left(s, t-\frac{r}{\varphi(r)}\right) d s+f(r, t)
$$

Where,

$$
\begin{gathered}
K(r, t, s)=\varphi^{-1}(r) \varphi(0) v\left(t-\frac{r}{\varphi(r)}\right) \beta\left(t-\frac{r}{\varphi(r)}\right) \mu\left(s, t-\frac{r}{\varphi(r)}\right) \\
\cdot \exp \left[-\int_{0}^{t} \mu(\varphi(r) \tau+r-\varphi(r) t, \tau) d \tau\right], \\
f(r, t)=\varphi^{-1}(r) \varphi(r-\varphi(r) t) p_{0}(r-\varphi(r) t) \\
\cdot \exp \left[-\int_{0}^{t} \mu(\varphi(r) \tau+r-\varphi(r) t, \tau) d \tau\right] .
\end{gathered}
$$

So the existence and uniqueness of the solutions of system (1) is equivalent to that of integral equation (5), we only need to prove that the solution of the integral equation (5) is existence and uniqueness.

We define operator as follows:

$$
\begin{gathered}
\mathrm{A}: C[0, T] \rightarrow C\left(\left[0, Q_{m}\right] \times[0, T]\right) \\
\mathrm{A} p(s, t)=p\left(s, t-\frac{r}{\varphi(r)}\right)
\end{gathered}
$$

Easy to know $\|\mathrm{A}\|=1$, so $\mathrm{A}$ is a bounded linear operator.

To define the integral operator

$$
\begin{aligned}
& \mathrm{K}: C[0, T] \rightarrow C[0, T] \\
& \mathrm{K} p(r, t)=\int_{0}^{\varphi(r) t} K(r, t, s) \mathrm{Ap}(s, t) d s
\end{aligned}
$$

So the integral equation (5) can be represented the form of operator equation as the following:

$$
p(r, t)=\mathrm{K} p(r, t)+f(r, t)
$$

From the assumptions (H), we know that $K(r, t, s)$ is bounded and continuous, so there is constant $M_{1}$, making $|K(r, t, s)| \leq M_{1}$, for $\varphi(r)$ is a Continuous and bounded function, so there is constant $M_{2}$, making $|\varphi(r)| \leq M_{2}$. so ,we can get the following results:

$$
\begin{aligned}
|\mathrm{K} p(r, t)|= & \left|\int_{0}^{\varphi(r) t} K(r, t, s) \mathrm{A} p(s, t) d s\right| \leq \int_{0}^{\varphi(r) t}|K(r, t, s) \mathrm{A} p(s, t)| d s \\
& \leq M_{1}\|p\| \int_{0}^{\varphi(r) t} d s \leq M_{1}\|p\| \varphi(r) t \leq M_{1} M_{2}\|p\| t \leq M_{1} M_{2} \| p \\
\left|\mathrm{~K}^{2} p(r, t)\right|= & \left|\int_{0}^{\varphi(r) t} K(r, t, s) \mathrm{AK} p(s, t) d s\right| \\
& \leq \int_{0}^{\varphi(r) t}|K(r, t, s) \mathrm{AK} p(s, t)| d s \leq M_{1}\left|\int_{0}^{\varphi(r) t} \mathrm{~K} p(s, t) d s\right| \\
& \leq M_{1}^{2}\|p\| \int_{0}^{\varphi(r) t} \varphi(r) s d s \leq M_{1}^{2}\|p\| \frac{\varphi^{2(2+1) / 2}}{2 !} t^{2} \\
& \leq M_{1} \frac{M_{2}^{2(2+1) / 2}}{2 !}\|p\| t^{2} \leq M_{1} \frac{M_{2}^{2(2+1) / 2}}{2 !}\|p\| T^{2},
\end{aligned}
$$

By mathematical induction, we can get the following general form:

$$
\begin{aligned}
\left|\mathrm{K}^{n} p(r, t)\right| & \leq M_{1}{ }^{n}\|p\| \frac{\varphi^{n(\mathrm{n}+1) / 2}}{n !} t^{n} \\
& \leq M_{1}{ }^{n} \frac{M_{2}{ }^{n(\mathrm{n}+1) / 2}}{n !}\|p\| t^{n} \\
& \leq M_{1}{ }^{n} \frac{M_{2}{ }^{n(\mathrm{n}+1) / 2}}{n !}\|p\| T^{n}
\end{aligned}
$$

So, the following formula is established

$$
\left\|\mathrm{K}^{n}\right\| \leq M_{1}^{n} \frac{M_{2}^{n(n+1) / 2}}{n !} T^{n}
$$

For $M_{1}, M_{2}, T$ are constant, when $n$ is large enough, $\left\|\mathrm{K}^{n}\right\| \leq M_{1}^{n} \frac{M_{2}^{n(n+1) / 2}}{n !} T^{n}<1$ is established. so the spectral radius of the operator is $\gamma_{\sigma}(\mathrm{K})=\lim _{n \rightarrow \infty} \sqrt[n]{\left\|\mathrm{K}^{n}\right\|}<1$, so $(\mathrm{I}-\mathrm{K})^{-1}$ is existence.

So we can obtain the solution of integral equation (5) as following form:

$$
p(r, t)=(\mathrm{I}-\mathrm{K})^{-1} f(r, t)
$$

From Hypothetical conditions, we know $f(r, t)$ is continuous, so (6) is the only solution of integral equation (5). So we can get the following theorem:

Theorem 1 Assume that the forest development system (1) satisfies the condition $(\mathrm{H})$, Then the positive solutions of system (1) is existence and uniqueness, And the solutions $p(r, t)$ can be represented by (2) and (6).

\section{STABILITY ANALYSIS}

Here we discussed the stability of the system (1) mainly by constructing Lyapunov function.

When growth function $\varphi(r)$ is differentiable, the equivalent form of forest development system (1) is as follows:

$$
\begin{array}{ll}
\frac{\partial p(r, t)}{\partial t}+\varphi(r) \frac{\partial p(r, t)}{\partial r}=-\mu(r, t) p(r, t)-g(r, t), & t, r>0 \\
p(r, 0)=p_{0}(r), & r \geq 0 \\
p(0, t)=v(t) \beta(t) \int_{0}^{Q_{m}} \mu(r, t) p(r, t) d r, & t \geq 0
\end{array}
$$

$p \| T$, Lemma $\mathbf{1}^{[8]}$ (Lyapunov stability theorem) If there is a positive definite function denoted by $V(x)$ in some areas, when $\left.\frac{d V}{d t}\right|_{(7)} \leq 0$, the solution of system (7) is stable; when $\left.\frac{d V}{d t}\right|_{(7)} \leq \delta<0$, Solution of system (7) is asymptotically stable, where $\delta$ is any constant which is less than zero.

According to the Lyapunov stability theory, by constructing Lyapunov function, to analyze the stability of the system, we can get the following conclusion.

Theorem 2 (the stability conditions of forest development system ) If the Lyapunov function is selected for $V(p)=\int_{0}^{Q_{m}} \varphi^{-1}(r) p^{2}(r, t) d r+2 \int_{0}^{Q_{m}}\left(\int_{0}^{t} \varphi^{-1}(r) p(r, s) g(r, s) d s\right) d r$, 
So when $(v(t) \beta(t))^{2} \int_{0}^{Q_{m}} \mu^{2}(r, t) d r-2 \mu_{0} \leq 0$, the solution of system (7) is stable; when $(v(t) \beta(t))^{2} \int_{0}^{Q_{m}} \mu^{2}(r, t) d r-2 \mu_{0} \leq \delta<0$, Solution of system (7) is asymptotically stable, where is any constant which is less than zero and $\mu_{0}=\inf _{(r, t) \in\left[0, Q_{m}\right] \times[0, T]}\left[\varphi^{-1}(r) \mu(r, t)\right]$.

Proof: Lyapunov function is selected for $V(p)=\int_{0}^{Q_{m}} \varphi^{-1}(r) p^{2}(r, t) d r+2 \int_{0}^{Q_{m}}\left(\int_{0}^{t} \varphi^{-1}(r) p(r, s) g(r, s) d s\right) d r$

Obviously $V(p)>0$, so $V(p)$ is a positive definite function.

When calculating directly, we get the results as follows:

$$
\begin{aligned}
\left.\frac{d V}{d t}\right|_{(7)}= & \int_{0}^{Q_{m}} \varphi^{-1}(r) \cdot 2 p(r, t) \cdot p_{t}(r, t) d r+2 \int_{0}^{Q_{m}} \varphi^{-1}(r) p(r, t) g(r, t) d r \\
= & \int_{0}^{Q_{m}} \varphi^{-1}(r) \cdot 2 p(r, t) \cdot\left[p_{t}(r, t)+g(r, t)\right] d r \\
= & \int_{0}^{Q_{m}} \varphi^{-1}(r) \cdot 2 p(r, t) \cdot\left[p_{t}(r, t)-\mu(r, t) p(r, t)\right. \\
= & \int_{0}^{Q_{m}} \varphi^{-1}(r) \cdot 2 p(r, t) \cdot\left[-\mu(r, t) p(r, t)-\varphi(r) p_{r}(r, t)\right] d r \\
= & -\int_{0}^{Q_{m}} \varphi^{-1}(r) \cdot 2 p^{2}(r, t) \cdot \mu(r, t)+2 p(r, t) p_{r}(r, t) d r \\
= & \int_{0}^{Q_{m}} \exp \left[-2 \int_{0}^{r} \varphi^{-1}(\tau) \mu(\tau, t) d \tau\right] \\
= & p^{2}(0, t)-\int_{0}^{Q_{m}} \varphi^{-1}(r) \cdot 2 p^{2}(r, t) \cdot \mu(r, t) d r \\
= & (v(t) \beta(t))^{2} \cdot\left(\int_{0}^{Q_{m}} \mu(r, t) p(r, t) d r\right)^{2} \\
& -\int_{0}^{Q_{m}} \varphi^{-1}(r) \cdot 2 p^{2}(r, t) \cdot \mu(r, t) d r \\
\leq & (v(t) \beta(t))^{2} \cdot \int_{0}^{Q_{m}} \mu^{2}(r, t) d r \cdot \int_{0}^{Q_{m}} p^{2}(r, t) d r \\
& -\int_{0}^{Q_{m}} \varphi^{-1}(r) \cdot 2 p^{2}(r, t) \cdot \mu(r, t) d r \\
= & \int_{0}^{Q_{m}}\left[(v(t) \beta(t))^{2} \int_{0}^{Q_{m}} \mu^{2}(r, t) d r-2 \varphi^{-1}(r) \mu(r, t)\right] \cdot p^{2}(r, t) d r \\
\leq & \int_{0}^{Q_{m}}\left[(v(t) \beta(t))^{2} \int_{0}^{Q_{m}} \mu^{2}(r, t) d r-2 \mu_{0}\right] \cdot p^{2}(r, t) d r .
\end{aligned}
$$

Where $\mu_{0}=\inf _{(r, t) \in\left[0, Q_{m}\right] \times[0, T]}\left[\varphi^{-1}(r) \mu(r, t)\right]$.

So from the Lyapunov stability theory.we can know when $(v(t) \beta(t))^{2} \int_{0}^{Q_{m}} \mu^{2}(r, t) d r-2 \mu_{0} \leq 0 \quad,\left.\quad \frac{d V}{d t}\right|_{(7)} \leq 0 \quad$, the solution of system (7) is stable and when $(v(t) \beta(t))^{2} \int_{0}^{Q_{m}} \mu^{2}(r, t) d r-2 \mu_{0} \leq \delta<0,\left.\frac{d V}{d t}\right|_{(7)} \leq \delta<0$, Solution of system (7) is asymptotically stable, where $\delta$ is any constant which is less than zero.
IV. DISCUSSING THE EXISTENCE AND UNIQUENESS OF THE SOLUTIONS BY USING THE OPERATOR SEMIGROUP THEORY

Reference [1] has discussed the properties of the solutions of stationary forest evolution equations by using semigroup theory, we can study the existence and uniqueness of solution of non-stationary forest evolution equations by defining time-varying operator and using the methods of semigroup theory in reference [7].

Set $X$ is a Banach space, defining the operator $A(t)$ on $X$ as following:

$$
\begin{gathered}
D(A(t))=\left\{p \in X \mid-\varphi^{\frac{1}{2}}(r) \frac{d}{d r}\left[\varphi^{\frac{1}{2}}(r) p(r)-\mu(r, t) p(r)\right] \in X\right. \\
\left.p(0)=\varphi^{\frac{1}{2}}(0) \nu(t) \beta(t) \int_{0}^{Q_{m}} \mu(r, t) p(r) d r, \forall t\right\} \\
A(t) p(r)=-\varphi^{\frac{1}{2}}(r) \frac{d}{d r}\left[\varphi^{\frac{1}{2}}(r) p(r)-\mu(r, t) p(r)\right], \quad p \in D(A(t)) .
\end{gathered}
$$

From reference [1], we can get the following two properties of operator $A(t)$ for arbitrary $t \in[0, T]$ :

(i) $A(t)$ is a dense and closed linear operator for arbitrary $t$ :

(ii) $A(t)$ Generated $C_{0}$ semigroup for arbitrary $t$.

So the system (1) can be represented by the form of the following initial value problem:

$$
\left\{\begin{array}{l}
\frac{d p(\mathrm{t})}{d t}=A(\mathrm{t}) p(\mathrm{t}) \\
p(0)=p_{0}
\end{array}\right.
$$

So we only need to discuss the existence and uniqueness of solution of system (8), the initial condition of System (8) is $t=0$, we put special into general, discussing the existence and uniqueness of solutions of the system at an arbitrary initial time $s \in[0, t]$. Then the general situation is easy to draw, rewriting the system (8) for the following form:

$$
\left\{\begin{array}{l}
\frac{d p(\mathrm{t})}{d t}=A(\mathrm{t}) p(\mathrm{t}) \\
p(\mathrm{~s})=g(\mathrm{~s}),
\end{array}\right.
$$

At this point we can convert to use the semigroup theory methods in literature [7] to discuss the existence and uniqueness of initial value problem (9), while the existence and uniqueness of solution of the original system was also proved.

\section{ACKNOWLEDGMENT}

The work is supported by the National Science Foundation of China (61273016).

\section{REFERENCES}

[1] Yu .J.Y, Wang .W.W, Zhu .G.T, properties of the solution of evolution for an age-structured forest square[J], Acta Mathematicae Applicatae Sinica,1994, 17(4):606-612.

[2] Wang .D.J, Zang.Y.F, The property of solution of a nonstationry forest evolution system[J].Systems science and Systems Engineering,1993, 2(3):78-87.

[3] Zhang .Q.M,Nie .Z.K, the exitence and uniqueness of strong solution of forest evolution system and the stability[J], Journal of Biomathematics, 2004,19(1):51-56. 
[4] Wang .D.J, existence and uniqueness of solution of nonlinear agestructured population evolution equations $[\mathrm{J}]$, Journal of Biomathematics, 1994,9(2):70-73.

[5] Wang .D.J, research development of nonlinear forest evolution systems[J], Journal of Biomathematics, 2008,23(1):125-131.

[6] Wang .D.J, the property of the solution of a nonlinear population evolution equation [J], Applied Mathematics A Journal of Chinese Universities, 1998,13(1):23-30.
[7] Li .Y.B, the application foundation of bounded linear operator semigroup $[\mathrm{M}]$, Liao ning : Liaoning Science and Technology Press, 1992.

[8] Liao .X.X, theory methods and application of stability[M], Wuhan : Huazhong university of science and technology press, 2010 . 